\title{
ANTIBIOTIC SUSCEPTIBILITY PATTERN OF STAPHYLOCOCCUS AUREUS WITH SPECIAL REFERENCE TO METHICILIN RESISTANCE - A PRELIMINARY REPORT
}

\section{ABSTRACT}

One hundred strains of Staphylococcus aureus isolated from various clinical samples were screened for methicillin resistance by the disc diffusion technique. Antibiotic susceptibility pattern of Methicillin sensitive S. aureus (MSSA) and Methicillin resistant S. aureus (MRSA) against a number of antibiotics like penicillin, ampicillin, gentamicin, erythromycin, tetracycline, Coamoxyclav cotrimoxazole, ciprofloxacin, cefalothin and vancomycin are compared. $20 \%$ of the strains were found to be MRSA while $80 \%$ were MSSA. The isolation rate of MRSA from indoor (hospital acquired) and outdoor (community acquired) was $18 \%$ and $2 \%$ respectively. Drug resistance of MRSA was highest with penicillin (100\%), followed by erythromycin (80\%), ampicillin and gentamicin $(65 \%)$, tetracycline $(60 \%)$, cotrimoxazole $(55 \%)$, cefalothin $(20 \%)$, Coamoxyclav (15\%) and least against ciprofloxacin (5\%) and vancomycin $(0 \%)$. The MSSA were sensitive to vancomycin $(\mathbf{1 0 0 \%})$, ciprofloxacin $(\mathbf{1 0 0 \%})$, Coamoxyclav $(96.25 \%)$, and cephalothin $(82.5 \%)$ while sensitivity to tetracycline $(\mathbf{7 8 . 7 5 \%})$, gentamicin $(75 \%)$, erythromycin $(40 \%)$, ampicillin $(38.75 \%)$, cotrimoxazole $(37.55)$ and penicillin $(\mathbf{2 1 . 2 5 \%})$ was much lesser. This study emphasizes the need for constant monitoring on the prevalence of MRSA and its microbial susceptibility pattern as the data would help clinicians in the effective management of nosocomial infections caused by S.aureus.

Key Words: Staphylococcus Aureus, Methicillin, Sensitive, Resistant, Nosocomial Infection.

1. Assistant Professor, Department of Microbiology, Durga Nursery Main Road, Udaipur, Rajasthan, India.

2. Tutor, Dept. of Microbiology, Manipal College of Medical Sciences, Pokhara, Nepal.

Address for correspondence : $\quad$ Dr. Chitra Pai, Assistant Professor, Department of Microbiology

12 E Shiva Park Colony, Durga Nursery Main Road

Udaipur, Rajasthan, India. 313001.

Email: paichitra@yahoo.com 


\section{INTRODUCTION}

Staphylococcus aureus has over the years proved its might as the most versatile and notorious pathogen. It has maintained its role as one of the commonest human pathogens in both community acquired and hospital acquired infections. It is also a notorious pathogen in producing a wide range of infections, more diverse than most other bacterial genera. Drug resistance in $\mathrm{S}$. aureus has been a major problem over the years. Strains resistant to a variety of drugs, 'multiresistant' strains are commonly present in hospitals and may present a difficulty in therapy. ${ }^{1}$ Over $90 \%$ of both hospital and community strains of $\mathrm{S}$. aureus causing infection are resistant to penicillin. ${ }^{2}$ With the introduction of methicillin in the nineteen sixties, a new resistant strain of S.aureus called Methicillin Resistant Staphylococcus aureus (MRSA) was discovered. MRSA have been reported worldwide and have been found to be responsible for outbreaks of nosocomial infections. ${ }^{3}$ Some strains associated with outbreaks are called Epidemic MRSA or EMRSA because once introduced they have a remarkable ability to spread within a hospital. ${ }^{4,5}$ Several hospitals have begun screening for MRSA, since infections caused by these strains significantly affect patient's morbidity and the hospital's reimbursement. Although it was previously thought that staphylococci are sensitive to vancomycin, ${ }^{6}$ recent reports of reduced susceptibility to vancomycin in some MRSA isolates has been rather disturbing. ${ }^{7,8,9}$

\section{This study was designed with the following} objectives:

1. To study the prevalence of methicillin resistance among the strains of $\mathrm{S}$. aureus

2. To determine the antibiotic susceptibility patterns of MRSA and MSSA strains against a panel of antibiotics.

\section{MATERIALS AND METHODS}

A hundred strains of Staphylococcus aureus isolated from various clinical samples received at the Clinical Microbiology Laboratory of Manipal Teaching Hospital, Pokhara were included in the study. Identification of S. aureus was based on morphology, colony characteristics and biochemical tests like catalase and coagulase tests. Antibiotic susceptibility tests of the strains were carried by the Kirby Bauer disc diffusion method. The antibiotics tested were penicillin (10 units), ampicillin (1Omcg), gentamicin (1Omcg), erythromycin $(15 \mathrm{mcg})$, tetracycline $(30 \mathrm{mcg})$, Coamoxyclav (30mcg), cotimoxazole $(25 \mathrm{mcg})$, ciprofloxacin $(5 \mathrm{mcg})$, cefalothin $(30 \mathrm{mcg})$, and vancomycin (30mcg). Methicillin susceptibility was carried out using methicillin disc $(5 \mathrm{mcg})$ placed on Mueller Hinton agar incubated at $30^{\circ} \mathrm{C}$ as well as Mueller Hinton agar supplemented with $6 \% \mathrm{NaCI}$ incubated at $37^{\circ} \mathrm{C}$ as per standard procedures. ${ }^{1}$ A standard strain of S. aureus ATCC 25923 was also included in the antibiotic susceptibility tests.

\section{RESULTS}

Out of the total of 100 strains of S. aureus included in the present study, 68\% strains were obtained from pus samples, $12 \%$ from blood, $10 \%$ from urine, $6 \%$ from sputum and $4 \%$ from throat swabs. Of the pus samples, majority were from cases of wound infections (32) and pyoderma(26) followed by breast abscess(5), ear discharge (4) and a case of tropical pyomyositis (1).

$20 \%$ of the strains were found to be MRSA while $80 \%$ were MSSA. Out of the 20 MRSA strains, 18 were isolated from pus samples, one from sputum and one was a urinary isolate. 18 of the 20 patients from whom MRSA were isolated, were inpatients while only 2 patients were those attending the OPD. 
These 18 patients had undergone surgery and had developed hospital acquired wound infection. Therefore the overall isolation rate of MRSA from indoor (hospital acquired) and outdoor (community acquired) was $18 \%$ and $2 \%$ respectively.

The results of antibiotic susceptibility patterns of the isolates are given in table I.

\section{Table 1}

\begin{tabular}{|l|c|c|c|c|c|}
\hline \multirow{2}{*}{ DRUG } & DISC & \multicolumn{2}{c|}{ MSSA $(\mathbf{n = 8 0})$} & \multicolumn{2}{c|}{ MRSA (n=20) } \\
\cline { 3 - 6 } & $\begin{array}{c}\text { CONC. } \\
(\boldsymbol{\mu g} \text { /DISC) }\end{array}$ & $\begin{array}{c}\text { No. } \\
\text { Sensitive }\end{array}$ & $\begin{array}{c}\text { \% } \\
\text { sensitive }\end{array}$ & $\begin{array}{c}\text { No. } \\
\text { Sensitive }\end{array}$ & $\begin{array}{c}\text { \% } \\
\text { sensitive }\end{array}$ \\
\hline PENICILLIN & 10 units & 17 & 21.25 & 0 & 0 \\
\hline AMPICILLIN & 10 & 31 & 38.75 & 7 & 35 \\
\hline GENTAMICIN & 15 & 60 & 75 & 7 & 35 \\
\hline ERYTHROMYCIN & 30 & 32 & 40 & 4 & 20 \\
\hline TETRACYCLINE & 30 & 63 & 78.75 & 8 & 40 \\
\hline AUGMENTIN & 30 & 77 & 96.25 & 17 & 85 \\
\hline COTRIMOXAZOLE & 25 & 30 & 37.5 & 9 & 45 \\
\hline CIPROFLOXACIN & 30 & 80 & 100 & 19 & 95 \\
\hline CEFALOTHIN & 30 & 66 & 82.5 & 16 & 80 \\
\hline VANCOMYCIN & 30 & 80 & 100 & 20 & 100 \\
\hline
\end{tabular}

\section{A COMPARISON OF ANTIBIOTIC} SUSCEPTIBILITY PATTERNS OF MRSA STRAINS WITH MSSA STRAINS.

\section{DISCUSSION}

'Staphylococcus aureus' the bunch of cocci has always proved to be sour for microbiologists and clinicians alike. Our study highlights the problem of drug resistance among the isolates of $\mathrm{S}$. aureus and also the prevalence of MRSA, which is about $20 \%$. This correlates well with the findings of Harish and coworkers. ${ }^{10}$ Mathur et al from Lucknow have reported an isolation rate of $33.5 \%,{ }^{11}$ while a surveillance study of MRSA by Mehta et al from Mumbai showed an isolation rate ranging from $31.8 \%$ to $36.8 \% .{ }^{12}$ The percentage of hospitals isolating MRSA in the west has increased from $2 \%$ in '70's to $30 \%$ in ' 90 's. ${ }^{13}$

In the present study, isolation of MRSA from hospitalised patients was quite high $(18 \%)$ as compared to isolation of MRSA from outdoor patients (2\%). The higher prevalence rate of MRSA in hospitalised patients might be due to prolonged surgery, a long stay in the hospital, instrumentation and / or invasive procedures. Besides, the role of hospital personnel as carriers also needs a special mention because many outbreaks of MRSA infections in hospitals have been traced to hospital personnel. ${ }^{14}$

MRSA has emerged as one of the most important nosocomial pathogens and multidrug resistance is a common feature of MRSA. ${ }^{15,16}$ This has been reiterated in our studies which shows that drug resistance of MRSA was highest with penicillin $(100 \%)$, followed by erythromycin $(80 \%)$, ampicillin and gentamicin $(65 \%)$, tetracycline $(60 \%)$, cotrimoxazole $(55 \%)$, cefalothin $(20 \%)$, Coamoxyclav ( $5 \%$ ) and least against ciprofloxacin $(5 \%)$ and vancomycin $(0 \%)$.

In comparison with the MRSA, the MSSA strains were more sensitive to various antibiotics as is evident from Table I. All the 80 strains were sensitive to ciprofloxacin and vancomycin, while many were resistant to penicillin and ampicillin. It is a well-established fact that most strains of $\mathrm{S}$. aureus, even those acquired in the community are penicillin resistant. In most cases this resistance is attributable to plactamase production due to genes located on extra chromosomal plasmids. ${ }^{17}$ Though all the strains of MSSA were sensitive to ciprofloxacin, 5\% of MRSA were resistant to it. Decrease in susceptibility to ciprofloxacin has also been reported in other studies though to a greater extent. ${ }^{10,12}$ Widespread use of ciprofloxacin even for simple infections in several hospitals has probably resulted in the emergence of resistant strains.

Susceptibility to vancomycin was $100 \%$ in both MSSA as well as MRSA strains. Though vancomycin remains the drug of choice for the treatment of infections with MRSA, recent reports of reduced susceptibility to vancomycin in some MRSA isolates have been rather disturbing. ${ }^{7,8,9}$ 
Our study emphasizes the fact that the problem of MRSA looms large on us. There is a need for constant monitoring of its prevalence and antimicrobial susceptibility patterns as the data from such studies will help the clinicians in effective treatment of MRSA infections. However much needs to be done in evaluating the clinical importance of MRSA. Ongoing strategies to prevent nosocomial transmission include, vigorous attempts to identify infected or colonised patients as well as active screening of health care workers, treatment of wound sites and carrier sites with mupirocin ointment and cohorting of caregivers and patients. ${ }^{18}$

\section{REFERENCES}

1. Duguid JP . Staphylococcus. In: Collee JG, Duguid JP, Fraser AG, Marmion BP, ed Mackie and MoCartney Practical Medical Microbiology, Vol 2., 13th edition, Churchill Livingstone Co, Edinburgh, 19 89: 303-316.

2. Locksley RM. Staphylococcal infections. In: Fauci AS, Braunwald E, Isselbacher KJ., Eds Harrison's Principles of Intemal Medicine. Vol 1. B^edition, McGraw-Hill, Inc.NewYork, 1994: 915-922.

3. Zaman R, Dibb WL. Methicillin resistant Staphylococcus aureus (MRSA) isolated in Saudi Arabia: epidemiology and antimicrobial resistance patterns. Journal of Hospital Infection. 1994; 26: 297-300.

4. Graham DR. Epidemic neonatal gentamicinmethicillin resistant Staphy-lococcus aureus infection associated with nonspecific topical use of gent amicin. J Pediatr.1980; 97: 972-978.

5. Reboli AC, John JF, Levkoff AH. Epidemic methici 17 ingentamicin-resistant Staphy-lococcus aureus in a neonatal intensive care unit. Am J Dis Child .1989; 143:34-39.

6. Sheagren JN. Staphylococcus aureus, the persistent pathogen. N Eng J Med . 1984; 310: 1368-1373, 1437-1442.

7. Hiramatsu K, Aritaka N, Hanaki H et al. Dissemination in Japanese hospitals of strains of Staphylococcus aureus heterogeneously resistant to vancomycin. Lancet. 1997; 350: 1668-1671.
8. Ariza J, Pujol M, Cabo J et al. Vancomycin in surgical infections due to Methicill in -resistant Staphylococcus aureus with heterogeneous resistance to vancomycin. Lancet. 1999; 353: 1587-1588.

9. Sieradzki K, Roberts RB, Haber SW, Tomasz A. The development of vancomycin resistance in a patient with methicillin- resistant 5' . aureus infection. N Eng J Med. 1999; 340:517-523.

10. Harish BN, Umesh Kumar PM, Udaya Shankar C et al. Prevalence of Methicillin resistant Staphylococcus aureus in JIPMER hospital- a prel iminary report. Indian J. Med. Micrdbiol. 1997; $15(3)$ : 137-138.

11. Mathur SK, Singhal S, PrasadKN, KishoreJ etal. Prevalence of Methicil I in resistant Staphylococcus aureus (MRSA) in a tertiary care hospital. Indian J. Med. Microbiol. 1994; 12:96-101.

12. Mehta AP, Rodrigues $C$, Sheth $K$ et al. Control of Methicillin resistant Staphy-lococcus in a tertiary care centre- a five-year study. Ind J Med Microbiol. 1998; 16 (1) : 31-34.

13. Gordon J. Clinical significance of methicillin sensitive and methicillin resistant Staphylococcus aureus in UK hospital and the relevance of povidone iodine in their control. Postgrad Med J. 1993; 69, Suppi 3: S106S116.

14. Lacksley RM. Multiple antibiotic resistant Staphylococcus aureus : introduction, transmission and evolution ofNosocomial infections. Ann-Int. Med. 1982; 97: 317-324.

15. Duckworth GJ. Diagnosis and management of methicillin resistant Staphylococcus aureus infection. B.M.J. 1993; 307: 1049-1052.

16. Paul N and Ayyagari A. Drug resistance pattern of methicill in resistant Staphy-lococcus aureus. Ind. Paed. 1991; 28: 725.

17. Micrococcaceae: Staphylococci, Micrococci and Stomatococci. In: Baron EJ, Peterson LR, and Finegold SM eds. Bailey and Scott's Diagnostic Microbiology^edition. Mosby Co.321-332.

18. Wenzel RP, Nettleman MD, Jones RN and Pfaller MA. Methicil l in - resistant Staphy-lococous aureus: implications for the 1990 's and effective control measures. Am J. Med. 1991; 91 (suppl 3B) 22 IS. 